\title{
Epidemiology of Pseudomonas aeruginosa in Intensive Care Unit and Otolaryngology Department of a Tunisian hospital
}

\author{
Karim Ben Slama ${ }^{1}$, Skander Gharbi ${ }^{1}$, Ahlem Jouini ${ }^{1}$, Meriem Maarouf ${ }^{1}$, Chedlia Fendri ${ }^{2}$, \\ Abdellatif Boudabous ${ }^{1}$ and Maher Gtari ${ }^{1 *}$ \\ ${ }^{1}$ Laboratoire Microorganismes et Biomolécules Actives, Faculté de Sciences de Tunis, 2092 Tunis, Tunisia. \\ ${ }^{2}$ Laboratoire de Bactériologie, Hôpital la Rabta, Tunis, Tunisia. \\ Accepted 23 August, 2011
}

\begin{abstract}
Ninety four clinical isolates of Pseudomonas aeruginosa cultured from patients admitted to the Intensive Care Unit (ICU) $(\mathrm{N}=37)$ and Otolaryngology $(\mathrm{ORL})(\mathrm{N}=57)$ during one year $(2001-2002)$ at the Rabta hospital (Tunisia) have been investigated by using serotyping, pyocintyping, drug susceptibility, M13-PCR and PFGE typing. Result shown that most of the isolates at the ICU belonged to serotype 0:12 $(11 / 37)$ that showed high resistance to commonly used antimicrobials ( $\beta$-lactamins, aminosids, and quinolone) and a predominance for pyocinotype P10. Despite the frequent occurrence of identical serotypes and pyocinotypes, most of the isolates represent unique RAPD-M13 genotype (88/94). PFGE typing detected three distinct clusters amongst the 012 isolates, suggesting a clonal relatedness among multiresistant 012 isolates. This study illustrates the importance of phenotypic and genotypic epidemiological surveillance of predominant 012 serotype clones in such service in local hospital.
\end{abstract}

Key words: Pseudomonas aeruginosa, epidemiology, clonal diffusion, multidrug resistance.

\section{INTRODUCTION}

$P$. aeruginosa is an opportunistic pathogen that is able to cause severe invasive diseases in critically ill and immunocompromised patients (Deplano et al., 2005). It is a common pathogen in hospitals and particularly in intensive care units (ICU) and shows a high phenotypic diversity (Fonseca et al., 2007). Moreover, high rates of resistance to antibiotics and frequent multidrugresistance (MDR) are associated with nosocomial $P$. aeruginosa strains (Di Martino et al., 2002). It has been associated with sporadic or clustered cases of infection generally confined to single hospitalization units. In contrast, it has much more rarely been involved in large hospital outbreaks or interhospital spreads (Pellegrino et al., 2002; Pitt et al., 1989). Propagation of such clones proceeds through complex routes that may involve, for example, the hands of health care personnel,

\footnotetext{
*Corresponding author. E-mail: maher.gtari@fst.rnu.tn.
}

environmental reservoirs, medical equipment, or reagents. A European survey on the prevalence of nosocomial infection in ICU patients showed that $P$. aeruginosa was one of the most frequent pathogens isolated from $29 \%$ of ICU-acquired infections (Vincent, 2000). Therefore, genomic diversity may allow this bacterium to expand its pathogenic potential (Head and Yu, 2004). Infection epidemiology has been investigated by means of several phenotypic and genotypic methods (Fegan et al., 1991; Liu et al., 1983).

The present study aimed the investigation of phenotypic and molecular epidemiology of $P$. aeruginosa isolates in ICU and ORL ward of the Rabta hospital with special emphasis on multiresistant $P$. aeruginosa $0: 12$ isolates. The correlations between antibiotic resistant pattern, pyocinotypes, siderotypes, and RAPD-M13 profiles in relation to serotypes of the isolates were also investigated. PFGE typing was used to suspect clonal relatedness among O:12 serotype $P$. aeruginosa isolates in ICU. 
Table 1. Serotype frequency distribution in ICU and ORL wards.

\begin{tabular}{lcc}
\hline Serotype & ICU & ORL \\
\hline O:11 & 9 & 7 \\
O:16 & 1 & 2 \\
O:4 & 1 & 6 \\
O:9 & 0 & 4 \\
O:6 & 4 & 7 \\
O:10 & 1 & 5 \\
O:1 & 3 & 5 \\
O:3 & 2 & 2 \\
O:12 & 11 & 0 \\
O:7 & 0 & 1 \\
O:2 & 1 & 2 \\
O:14 & 0 & 1 \\
NAM & 4 & 11 \\
PAM & 0 & 1 \\
PAG & 0 & 3 \\
\hline
\end{tabular}

\section{MATERIALS AND METHODS}

\section{Bacterial strains}

During the period of September 2001 and September 2002, 94 isolates of $P$. aeruginosa were collected from patients admitted to the intensive care unit $(\mathrm{N}=37)$ and otolaryngology ward $(\mathrm{ORL})(\mathrm{N}=$ 57 ) of the Rabta Hospital. The isolates were initially characterized on the basis of colonial morphology, positive oxidase reaction and motility. They were identified as $P$. aeruginosa by conventional methods on the basis of their production of pyoverdine on King $B$ medium and other chemical characters in the API20NE system (BioMérieux, France).

\section{Serotyping}

Isolates were serotyped by slide agglutination according to the international serogrouping schema for $P$. aeruginosa (Head and $\mathrm{Yu}$, 2004; Liu et al., 1983).

\section{Antibiotic susceptibility}

Antimicrobial susceptibility testing was carried out on MüellerHinton agar by a standard agar diffusion method, using ticarcillin, ceftazidime, ciprofloxacin, piperacillin, tobramycin, amikacin, cefsulodin and imipenem. It was interpreted according to the guidelines of the CA-SFM (2008). For purposes of this study, resistance to at least three of the following four drugs (ceftazidime, imipenem, tobramycin, ciprofloxacin) was considered multidrug resistance (MDR).

\section{Pyocintyping}

This was carried out according to a previously described method (Fyfe et al., 1984). This method identifies 105 main types and 25 subtypes on the basis of different inhibition patterns observed against thirteen indicator strains.

\section{RAPD-M13 fingerprinting}

The genomic DNA was prepared using a rapid method for gramnegative bacteria (Chen and Kuo, 1993). RAPD-M13 fingerprinting was performed as previously described (Miteva et al., 1998). M13PCR products were analyzed by $2 \%$ agarose gel electrophoresis at $5 \mathrm{~V} / \mathrm{cm}$. The DNA molecular weight $100 \mathrm{bp}$ Ladder was used as a size standard. Positions of M13 patterns bands were transformed into a binary character matrix. Pairwise distance matrices were compiled using percent similarity. Phylogenetic tree was created by the UPGMA average cluster analysis using MVSP Version 3.1 (Kovach, 1999).

\section{PFGE}

PFGE typing was performed for eleven 012 serotype $P$. aeruginosa isolates. Genomic DNA from $P$. aeruginosa was prepared by the procedure described previously (Grundmann et al., 1995). In each case, the plug was digested with $20 \mathrm{U}$ Spe I restriction enzyme. PFGE was performed using a CHEF DRII system (Biorad). Running conditions consisted of two ramps in sequence (ramp A consisted of an initial switch time of $0.5 \mathrm{~s}$, a final switch time of $25 \mathrm{~s}$, and a run time of $20 \mathrm{~h}$; ramp B consisted of an initial switch time of $30 \mathrm{~s}$, a final switch time of $60 \mathrm{~s}$, and a run time of $4 \mathrm{~h}$ ). Gel pictures were interpreted using Gelpro 3.1 software for windows (media cybernetics). The peak positions of PFGE patterns were analysed. The UPGMA linkage was applied using MVSP Version 3.1. Potential clonal relatedness was determined at an $80 \%$ level of similarity corresponding to a maximum six-band difference (Goering and Tenover, 1997).

\section{RESULTS}

\section{Serotyping}

The $P$. aeruginosa isolates were O-serotyped immediately after isolation (Table 1). Serotyping generated an overall number of 12 different types. Remarkable differences in the incidence of individual O-serotypes were observed by comparing isolates from ICU and ORL Ward. Serotype O:12 was most common in ICU, representing $11 / 37(29.73 \%)$ of all ICU isolates. Most of the strains at the ORL ward belonged to serotypes O:11, O:6 $(7 / 57 ; 12.28 \%$ each) and $0: 4(6 / 57 ; 10.53 \%)$. Isolates with serotypes $0: 9,0: 7$ and $0: 14$ were detected at the ORL ward but they were absent at the ICU. Those with serotype 0:12 most represented in the ICU were absent at the ORL ward. Thus, only five major serotypes $0: 11$, O:6, O:12, O:1 and 0:4 were representative for 53/94 $(56.38 \%)$ of $P$. aeruginosa isolated in the Rabta hospital with predominance for O:11 serogroup (16/94; 17.02\%).

\section{Antibiotic susceptibility}

The results of drug susceptibility testing were shown in Figure 1. ORL ward isolates were highly susceptible to antimicrobials tested. $P$. aeruginosa isolates showed high resistance to commonly used antimicrobials especially in ICU. Antibiotic susceptibility results suggested that 


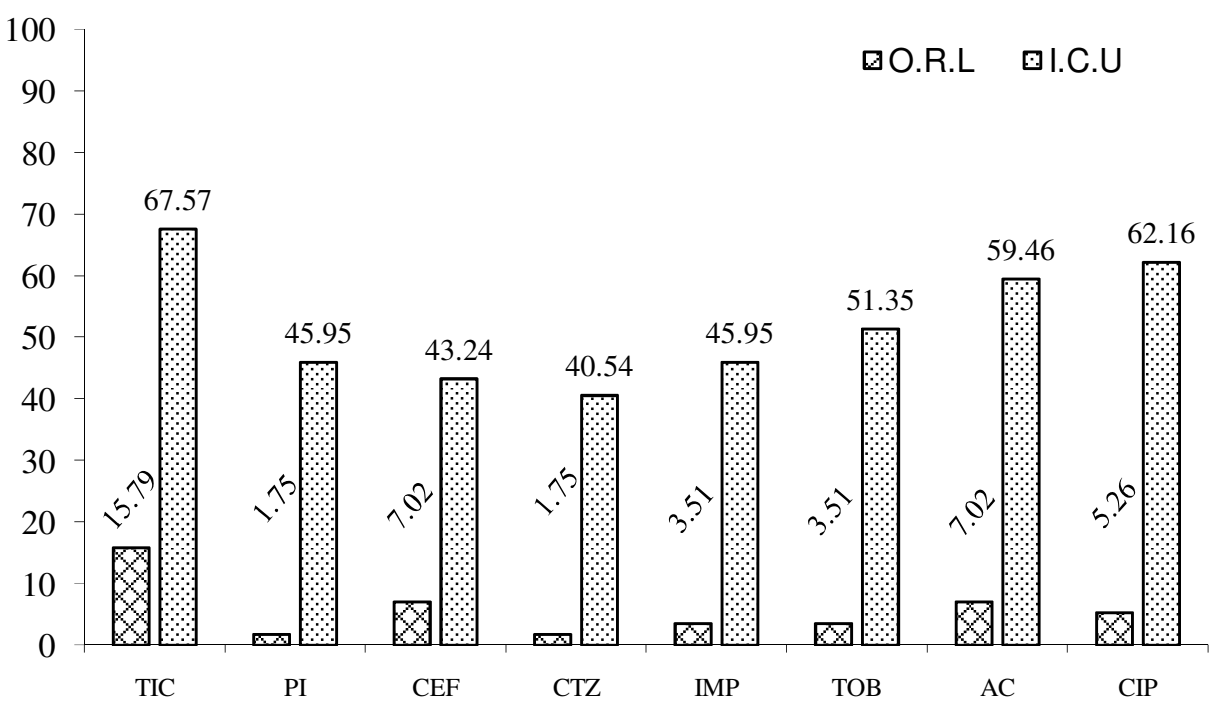

Figure 1. Resistance (\%) of $P$. aeruginosa isolated from ICU and ORL at Rabta hospital to various antibiotics. TIC, ticarcillin; PI, piperacillin; CEF, cefsulodin; CTZ, ceftazidime; IMP, imipenem; TOB, tobramycin; AC, Amikacin; CIP, ciprofloxacin.

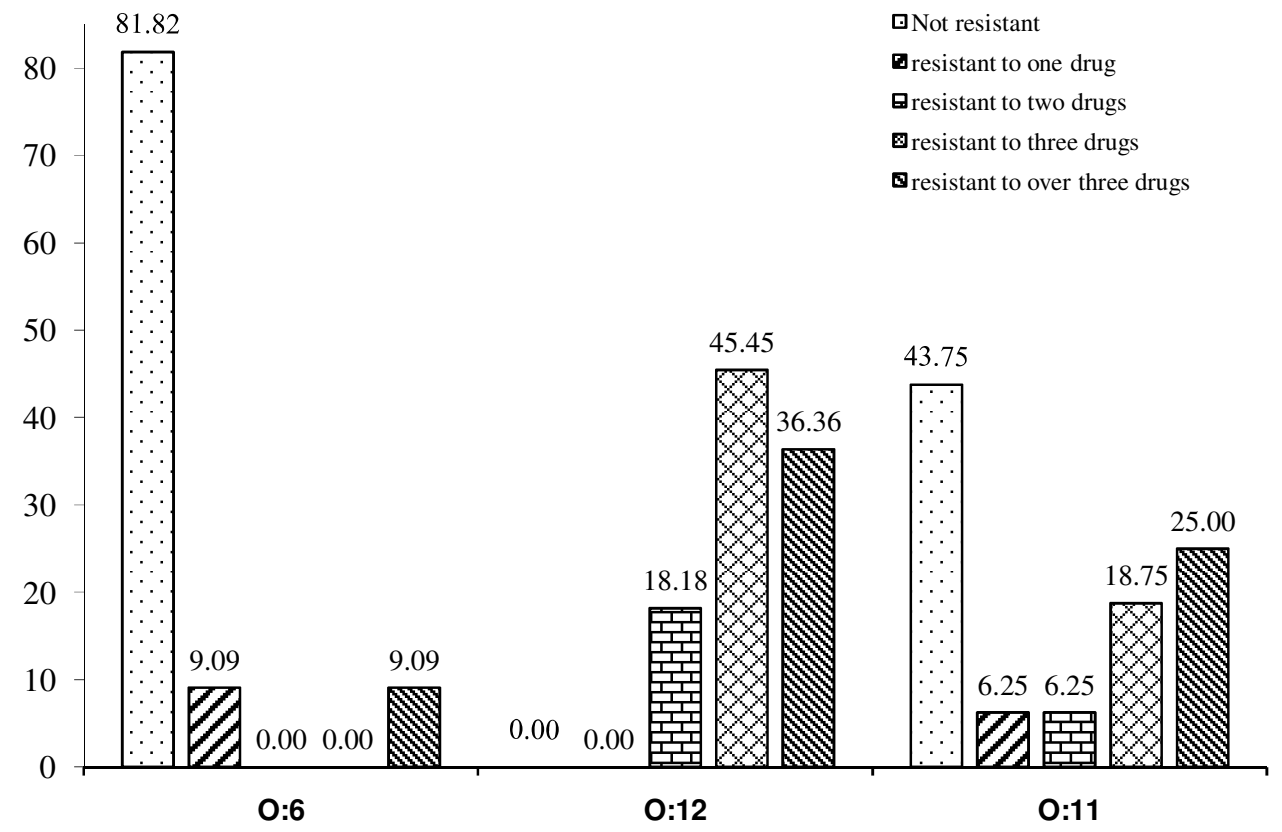

Figure 2. The number of anti-pseudomonal drugs (ceftazidime, imipenem, tobramycin, ciprofloxacin) to which predominant $P$. aeruginosa serotypes (O:6, O:12 and O:11) were

ceftazidime was the most effective to isolated strains.

To evaluate the extent of multidrug resistance, the number of individual drugs (ceftazidime, imipenem, tobramycin, ciprofloxacin) to which $P$. aeruginosa were resistant was assessed. ICU $P$. aeruginosa isolates were highly multidrug resistant compared to ORL ward isolates. Only (7/37) of ICU isolates were sensitive to all of this drugs and (18/37) were considered multidrug resistant. However, (36/57) ORL $P$. aeruginosa isolates were sensitive to all used drugs and no one was multidrug resistant (MDR). Analysis of antibiograms by serotype show the extend of multidrug resistance of the most prevalent serotypes (0:11, $0: 6$ and $0: 12$ ) as represented in Figure 2. The results show that susceptibility to all drugs used was represented by $9 / 11$ and $7 / 16$ respectively for $0: 6$ and $0: 11$ isolates. None O:12 serotype $P$. aeruginosa isolates were susceptible to all these drugs. Whereas, multidrug resistance was 
represented by $1 / 11,7 / 16$ and $9 / 11$ respectively for $0: 6$, $\mathrm{O}: 11$ and $\mathrm{O}: 12 \mathrm{P}$. aeruginosa isolates.

\section{Pyocintyping}

The $P$. aeruginosa isolates were subdivided into 21 pyocinotypes as shown in Figure 3 . The most represented pyocinotypes are P10 (41/94), P1 (5/94) and P105, P17 and P6 (3/94 each). The non-pyocinotypable (NT) isolates that produce pyocins with inhibition profile inexistent in the pyocinotypes table of Gillies and Govan (1966) are represented by $18 / 94$ isolates. The results show 1/94 atypical (AT) isolate with no pyocin production. Among the most represented pyocinotypes P10 and 2/41 are non-subpyocinotypables. Whereas, $39 / 41$ can be subdivided into 6 subpyocinotypes.

\section{RAPD-M13 fingerprinting}

A total of 90 different profiles were obtained by RAPDM13 fingerprinting. Most profiles (88/90) were unique, but 2 profiles were common each for 3 strains that were not necessarily from the same ward. The different $\mathrm{M} 13$ profiles were compared by numerical methods and the resultant dendrogram based on the similarity percentage between isolates, shows a high degree of genetic diversity as represented in Figure 3 . Seven major groups were defined at the $70 \%$ similarity level.

\section{PFGE typing}

Genomic relatedness of the eleven $012 P$. aeruginosa isolates was investigated by comparing the macrorestriction profiles of Spel-digested genomic DNA analyzed by PFGE. The obtained PFGE profiles were not identical but identify three cluster (designated I to III), suggesting clonal relatedness represented in Figure 4.

\section{DISCUSSION}

Serotyping of $P$. aeruginosa has been subject to several studies (Ben Slama et al., 2001; Bouhaddioui et al., 2002; Jerboui, 1997; Nour, 1990) that show the predominance in Tunisia of serotypes $0: 1,0: 6,0: 11$ and $0: 10$ at hospital environment. These serotypes dominated during the same period in European, Asiatic and African countries (Bert et al., 1994; Kezzal and Rahal, 1986; Shahcheraghi et al., 2003; Vanhoof et al., 1993; Vieu et al., 1987). Permanent investigation by serotyping allows us to suspect quickly epidemic dissemination when rarely encountered serotype appears within several patients of the same ward or the same hospital. The 0:12 serogroup was the most frequent in the ICU ward, contrary to previous studies reporting that $0: 12$ serogroup was absent or rarely encountered in Algeria $(0.25 \%)$, Mauritania (1.5\%) and in Tunisia (4.6\% or absent) (Ben Slama et al., 2001; Bouhaddioui et al., 2002). Its expansion seems to be caused by the multiple antibiotics resistances (Kezzal and Rahal, 1986; REUSSIR, 2001).

Thus, generally, serotype 0:12 proved to be the most resistant to antibiotics tested in the present study. Moreover, these results are in agreement with previous observations on the relatively frequent isolation of multiresistant serotype 0:12 strains in some European countries (Tzouvelekis et al., 1989), in Egypt (Vieu et al., 1984) and in the Indian sub-continent (Rukmini Devi and Bhaskaran, 1984). Finally, it should be pointed out that O:12 serotype strains predominant in ICU are associated with a particular virulent phenotype enhancing their ability to colonize the ICU patients and cause infection (REUSSIR, 2001). These strains were suspected of belonging to a single outbreak, suggesting nosocomial transmission of $P$. aeruginosa 0:12 and cross-infection within this ward.

The differences in proportion of antibiotic resistance between the two hospital wards investigated may be due to the application of different policies in treatment of patients or differences in the management of the ICU and ORL wards. This high resistance to antibiotics indicates the improper use of antibiotics at hospitals, as shown in many studies (Shahcheraghi et al., 2003; Struelens 1998).

$P$. aeruginosa pyocintyping has been the object of several previous studies. In Tunisia, the most predominant pyocinotypes between 1987/1988 were P1 $(40 \%)$ and P10 (30\%) (Bouhaddioui et al., 2002). In Europe, the same pyocinotypes (P1, P3, P5 and P10) were dominant at hospitals and environment with frequencies between 58 and $89 \%$ as previously reported (Orsi et al., 1994; Poh et al., 1988).

It is interesting to note that among the prevalent serotypes $0: 11,0: 12$ and $0: 6 \quad P$. aeruginosa, high genetic heterogeneity could be documented based on RAPD-M13 typing in accordance with a previous reports on clinical $P$. aeruginosa isolates (Fonseca et al., 2007; Morales et al., 2004).

The majority of the strains from serotype 0:12 are distributed by two major clusters. Moreover, these results indicate that RAPD-M13 typing can differentiate between the dominant multidrug resistant O:12 isolates in ICU as reported by (Elaichouni et al., 1994). The results of the present work also show that the phenotypic clusters of the isolates were not congruent with M13-PCR clonal lineages.

As a result, it can be assumed that clusterization by use of RAPD-M13 was poorly significant. Analysis of clinical isolates by other molecular techniques can be helpful in studying the epidemiology of outbreak strains, and in confirming their clonality (De Vos et al., 1997).

Comparing the PFGE fingerprints and antibiotic 


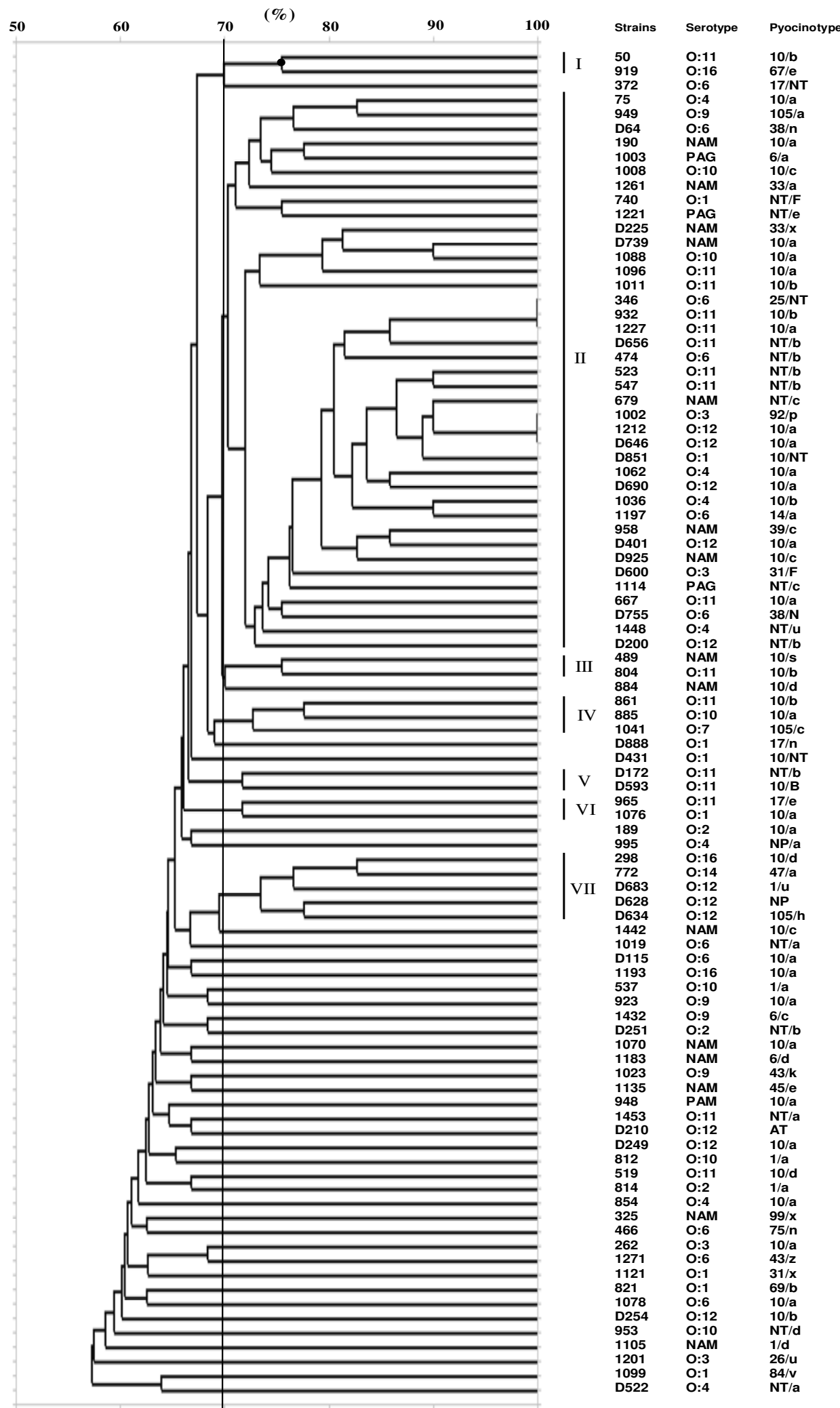

Figure 3. Computed dendrogram derived from digitized RAPD-M13 patterns for $94 P$. aeruginosa strains. The dendrogram was constructed by cluster analysis by UPGMA. The scale at the top of this figure shows the percentage similarity. The line in the dendrogram denotes the threshold $70 \%$ homology for defining groups of genetic similarity. Roman numerals indicate clusters with $\geq 70 \%$ homology. NT- nonpyocinotypable; AT- atypical. 


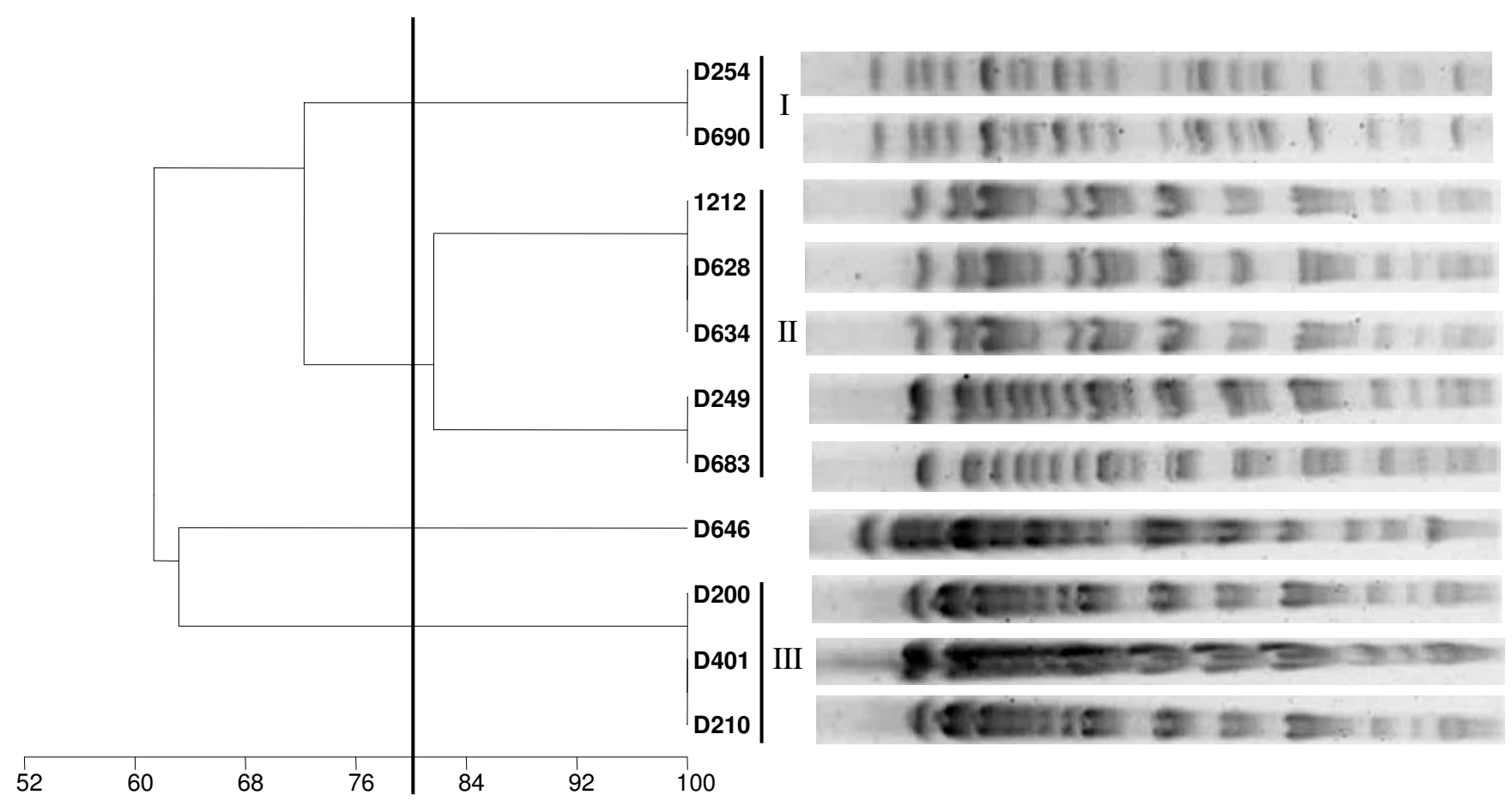

Figure 4. Pulsed Field Gel Electrophoresis banding patterns after Spel digestion of 012 serotype $P$. aeruginosa isolates. The dendrogram is based on a cluster analysis of the unweighted-pair group method with percent similarity. The cutoff at the $80 \%$ level of genetic similarity is indicated by a vertical line.

resistance patterns, the multiresistant $\mathrm{O} 12$ isolates are shown to be clonal (cluster II). PFGE analysis confirmed clonality for $\mathrm{O} 12 \mathrm{P}$. aeruginosa isolates between patients and over time. The extent of clonal dissemination within the hospital was estimated by determining the ratio of infected patients to clones. The results presented higher values in ICU of the Rabta hospital, between 2 to 5 ( 5 infected patients per clone), denoting the occurrence of epidemic or endemic infections with $012 P$. aeruginosa isolates during the study period.

These results were in agreement with previous studies, which showed that the 012 serotype $P$. aeruginosa has a long-recognized association with multiresistance and that a single MDR strain could persist in the same patient for many months and cause recurrent episodes of clinical infection (Hsueh et al., 1998; Nordmann and Poirel, 2002).

These findings are consistent with previous studies on Outbreaks of serotype $012 \mathrm{P}$. aeruginosa in Italy, Greece, and Korea showing their tight clonality (Crespo et al., 2004). It has been previously proposed, that the uniformity of characters of multiresistant $0: 12 P$. aeruginosa in Europe is suggestive of a common origin for these strains (Pitt et al., 1989). Previous finding, described the relatively high frequency of isolation of serotype 12 in Italy, showed to be resistant to a large number of antibiotics. They were in particular responsible for extensive and life-threatening outbreaks (Giammanco et al., 1985).

\section{Conclusion}

The present work showed a high phenotypic diversity of the studied clinical strains of $P$. aeruginosa. A specific cluster associated with multidrug resistant 012 serotype isolates, and PFGE genogroups was found suggesting the occurrence of epidemic or endemic infections with $012 P$. aeruginosa isolates during the study period. Thus, the results of the current study demonstrated that both the management and the policy of treatment should be revised at ICU and ORL wards at Rabta hospital in order to lower the burden of resistant bacteria from the environment. Such measures may also contribute to reducing the spread of resistance genes to other opportunistic microorganisms. This study illustrates the importance of phenotypic and genotypic surveillance to perform infection control. Indeed, $P$. aeruginosa strains have been recognized as a major public health problem, and therefore, active surveillance is needed to detect and prevent the dissemination of these $\mathrm{O} 12$ serotype epidemic clones.

\section{ACKNOWLEDGMENTS}

This study was funded by the Tunisian Ministry of Higher Education, Scientific Research and Technology grants (L206) to Microorganisms and Active Biomolecules Laboratory. 


\section{REFERENCES}

Ben Slama K, Boudabous A, Gharbi S, Cherif A, Chedlya F, Boelens H, Maaskant J, Verbrugh $H$, Van Belkum A (2001). Heterogeneity among infecting strains of $P$. aeruginosa in diverse departments of a large Tunisian hospital. J. Hosp. Infect., 47(4): 325-327.

Bert F, Bruneau B, Lambert-Zechovsky N, Branger C (1994). Etude épidémiologique de la sensibilité aux antibiotiques de Pseudomonas aeruginosa. Path. Biol., 42: 491-497.

Bouhaddioui B, Ben Slama K, Gharbi S, Boudabous A (2002). Epidemiology of clinical and environmental $P$. aeruginosa strains. Ann. Microbiol., 52: 223-235.

CA-SFM (2008). Comité de l'Antibiogramme de la Société Française de Microbiologie. Communiqué 2008.

Chen W, Kuo T (1993). A simple and rapid method for the preparation of Gram-negative bacterial genomic DNA. Nucleic Acids Res., 21: 2260.

Crespo MP, Woodford N, Sinclair A, Kaufmann ME, Turton J, Glover J, Velez JD, Castaneda CR, Recalde M, Livermore DM (2004). Outbreak of carbapenem-resistant $P$. aeruginosa producing VIM-8, a novel metallo-beta-lactamase, in a tertiary care center in Cali, Colombia. J. Clin. Microbiol., 42(11): 5094-5101.

De Vos D, Lim A, Pirnay JP, Duinslaeger L, Revets H, Vanderkelen A, Hamers R, Cornelis P (1997). Analysis of epidemic P. aeruginosa isolates by isoelectric focusing of pyoverdine and RAPD-PCR: modern tools for an integrated anti-nosocomial infection strategy in burn wound centres. Burns, 23(5): 379-386.

Deplano A, Denis O, Poirel L, Hocquet D, Nonhoff C, Byl B, Nordmann P, Vincent JL, Struelens MJ (2005). Molecular characterization of an epidemic clone of panantibiotic-resistant Pseudomonas aeruginosa. J. Clin. Microbiol., 43(3): 1198-1204.

Di Martino P, Gagniere H, Berry H, Bret L (2002). Antibiotic resistance and virulence properties of $P$. aeruginosa strains from mechanically ventilated patients with pneumonia in intensive care units: comparison with imipenem-resistant extra-respiratory tract isolates from uninfected patients. Microb. Infect., 4(6): 613-620.

Elaichouni A, Verschraegen G, Claeys G, Devleeschouwer M, Godard C, Vaneechoutte M (1994). P. aeruginosa serotype 012 outbreak studied by arbitrary primer PCR. J. Clin. Microbiol., 32(3): 666-671.

Fegan M, Francis P, Hayward AC, Fuerst JA (1991). Heterogeneity, persistence, and distribution of $P$. aeruginosa genotypes in cystic fibrosis patients. J. Clin. Microbiol. 29(10): 2151-2157.

Fonseca AP, Correia P, Sousa JC, Tenreiro R (2007). Association patterns of $P$. aeruginosa clinical isolates as revealed by virulence traits, antibiotic resistance, serotype and genotype. FEMS Immunol. Med. Microbiol., 51(3): 505-516.

Fyfe JA, Harris G, Govan JR (1984). Revised pyocin typing method for Pseudomonas aeruginosa. J. Clin. Microbiol., 20(1): 47-50.

Giammanco A, Di Stefano R, Arista S, Sinatra A, Chiarini A (1985). Infections caused by Pseudomonas aeruginosa: relatively frequent isolation of serogroup 12 from clinical specimens. Eur. J. Epidemiol., 1(2): 104-109.

Goering RV, Tenover FC (1997). Epidemiological interpretation of chromosomal macro-restriction fragment patterns analyzed by pulsed-field gel electrophoresis. J. Clin. Microbiol., 35(9): 2432-2433.

Grundmann H, Schneider C, Hartung D, Daschner FD, Pitt TL (1995). Discriminatory power of three DNA-based typing techniques for Pseudomonas aeruginosa. J. Clin. Microbiol., 33(3): 528-534.

Head NE, Yu H (2004). Cross-sectional analysis of clinical and environmental isolates of Pseudomonas aeruginosa: biofilm formation, virulence, and genome diversity. Infect. Immun., 72(1): 133-144.

Hsueh PR, Teng LJ, Yang PC, Chen YC, Ho SW, Luh KT (1998). Persistence of a multidrug-resistant $P$. aeruginosa clone in an intensive care burn unit. J. Clin. Microbiol., 36(5): 1347-1351.

Jerboui Z (1997). Etude des marqueurs épidémiologiques des souches de $P$. aeruginosa isolées en milieu hospitalier. Thèse de doctorat. Faculté des Science de Tunis: 135.
Kezzal K, Rahal K (1986). Pseudomonas aeruginosa. Application of serotyping and pyocin typing to strains isolated in Algeria. Arch. Inst. Pasteur., 55: 61-77.

Kovach W (1999). A Multivariate Statistical Package for Windows, ver 3.1. Kovach Computing Services, Pentraeth, UK.

Liu PV, Matsumoto H, Kusama H, Bergan T (1983). Survey of heatstable, major somatic antigens of Pseudomonas aeruginosa. Int. J. Syst. Bacteriol., 33: 256-264.

Miteva V, Gancheva A, Mitev V, Ljubenov M (1998). Comparative genome analysis of Bacillus sphaericus by ribotyping, M13 hybridization, and M13 polymerase chain reaction fingerprinting. Can. J. Microbiol., 44: 175-180.

Morales G, Wiehlmann L, Gudowius P, van Delden C, Tummler B, Martinez JL, Rojo $\mathrm{F}$ (2004). Structure of $P$. aeruginosa populations analyzed by single nucleotide polymorphism and pulsed-field gel electrophoresis genotyping. J. Bacteriol., 186(13): 4228-4237.

Nordmann P, Poirel L (2002). Emerging carbapenemases in gram negative aerobes. Clin. Microbiol. Infect., 8: 321-331.

Nour M (1990). Marqueurs épidémiologiques de $P$. aeruginosa isolés en milieu hospitalier., Thèse de Doctorat de spécialité. Faculté des Sciences de Tunis, p. 137.

Orsi GB, Mansi A, Tomao P, Chiarini F, Visca P (1994). Lack of association between clinical and environmental isolates of $P$. aeruginosa in hospital wards. J. Hosp. Infect., 27(1): 49-60.

Pellegrino FL, Teixeira LM, Carvalho G, Mda Md, Aranha Nouer S, Pinto De Oliveira M, Mello Sampaio JL, D'Avila Freitas A, Ferreira AL, Amorim Ed Ede L, Riley LW, Moreira BM (2002). Occurrence of a multidrug-resistant $P$. aeruginosa clone in different hospitals in Rio de Janeiro, Brazil. J. Clin. Microbiol., 40(7): 2420-2424.

Pitt TL, Livermore DM, Pitcher D, Vatopoulos AC, Legakis NJ (1989). Multiresistant serotype 012 Pseudomonas aeruginosa: evidence for a common strain in Europe. Epidemiol. Infect., 103(3): 565-576.

Poh CL, Yap EH, Tay L, Bergan T (1988). Plasmid profiles compared with serotyping and pyocin typing for epidemiological surveillance of Pseudomonas aeruginosa. J. Med. Microbiol., 25(2): 109-114.

REUSSIR (2001). Are the prolonged carrier and interhospital clonal diffusion of serotype $\mathrm{O} 12$ multiresistant $\mathrm{P}$. aeruginosa connected? Pathol. Biol., (Paris), 49(8): 620-623.

Rukmini Devi K, Bhaskaran CS (1984). Comparison of seroaeruginocine-phage typing of Pseudomonas aeruginosa. Ind. J. Pathol. Microbiol., 27(4): 281-288.

Shahcheraghi F, Feizabadi MM, Yamin V, Abiri R, Abedian Z (2003). Serovar determination, drug resistance patterns and plasmid profiles of $P$. aeruginosa isolated from burn patients at two hospitals of Tehran (IRAN). Burns, 29(6): 547-551.

Struelens M (1998). Stratégie d'investigation en cas d'épidémie à $P$. aeruginosa et Stenotrophomonas maltophilia. Méd. Mal. Infect., 28: 102-108.

Tzouvelekis LS, Tumah H, Malliara K, Legakis NJ (1989). Relationship of antibiotic resistance phenotype to the R-pyocin susceptibility pattern in clinical isolates of Pseudomonas aeruginosa. J. Chemother., 1(4): 226-230.

Vanhoof R, Godard C, Nulens E, Nyssen HJ, Wildemauwe C, Hubrechts JM, Maes P, Hannecart-Pokorni E (1993). Serotypes and extended spectrum beta-lactam resistance in aminoglycoside resistant $P$. aeruginosa isolates from two Belgian general hospitals: a seven year study. J. Hosp. Infect., 24(2): 129-138.

Vieu JF, Allos G, Hassan-Massoud B, Santos-Ferreira MO, Tselentis G (1984). Is there a geographic epidemiology of serogroups $O$ of Pseudomonas aeruginosa? Bull. Soc. Pathol. Exot. Filiales., 77(3): 288-294.

Vieu JF, Lepers JP, Chamoiseau G, Billon C, Klein B (1987). Epidemiology of $P$. aeruginosa in Mauritania: study of 239 strains of various origins. Bull. Soc. Pathol. Exot. Filiales, 80(5): 771-780.

Vincent $J L$ (2000). Microbial resistance: lessons from the EPIC study. European Prevalence of Infection. Intensive Care Med., 26(Suppl 1): S3-8. 